Zbl Arbeitsmed 2021 $\cdot 71: 49-55$ https://doi.org/10.1007/s40664-020-00411-9 Eingegangen: 6. Juli 2020

Angenommen: 25. September 2020

Online publiziert: 29. Oktober 2020

(c) Der/die Autor(en) 2020

Alexandra M. Preisser - Julia Pieter - Volker Harth

Zentralinstitut für Arbeitsmedizin und Maritime Medizin (ZfAM), Universitätsklinikum HamburgEppendorf, Hamburg, Deutschland

\title{
Präsenzlehre an Universitäten und Hochschulen unter den Bedingungen der SARS-CoV-2- Pandemie
}

\section{Empfehlungen zu technischen, organisatorischen und persönlichen Schutzmaßnahmen im Rahmen des Arbeitsschutzes}

\begin{abstract}
tient*innen-Kontakt und anderen Personen, welche als Risikopersonen angesehen werden müssen. Mindeststandard muss hier Schutzkleidung mit Kittel und medizinischer MundNasen-Schutz (MNS) für Studierende und Patient*innen sein.
\end{abstract}

Der Artikel richtet sich v. a. an Entscheidungsträger*innen und die für Arbeitsschutz und Hygiene verantwortlichen Personen in der Lehre an Universitäten und Hochschulen.

Die Empfehlung ist in weiten Teilen auch auf andere Formen der Erwachsenenbildung zu übertragen.

\section{Hintergrund}

Im Rahmen der nationalen Maßnahmen zur Verhinderung einer schnellen Ausbreitung der Infektion mit SARSCoV-2 haben die Länder ihre Universitäten und Hochschulen angewiesen, den Lehrbetrieb zu reduzieren und OnlineLehre anzubieten. Entsprechend des Einheitlichen Arbeitsschutzes gegen das Corona-Virus des Bundesministeriums für Arbeit und Soziales (BMAS) [1] sollen durch passende Maßnahmen des Arbeits- und Infektionsschutzes größere Menschenansammlungen vermieden und die Einhaltung der Abstandsregeln sichergestellt werden. Neben den klassischen Vorlesungen bietet die moderne Lehre an Hochschulen und Universitäten viele weitere Formate: Seminare, studentische Diskussionsgruppen, strukturierte Praktika in Versuchslaboren (MINTFächer ${ }^{1}$ ), Kleingruppenunterricht (im Betrieb, Krankenhaus), Selbststudium in Bibliotheken und E-Learning. Die Einhaltung der Abstandsregeln entsprechend des Arbeitsschutzstandards des BMAS [1] ist v. a. beim Aufeinandertreffen von Studierenden und Lehrkräften in großen Gruppen (Hörsaal) und kleinen Lehrräumen (Seminare, Praktika) zu ermöglichen. Besonders herausfordernd ist die Einhaltung des Infektionsschutzes bei sehr engem Abstand, evtl. sogar Körperkontakt untereinander (Schauspiel und Tanz, medizinische Kurse), oder mit Proband ${ }^{\star}$ innen und Patient ${ }^{\star}$ innen, die nicht Beschäftigte der Hochschule sind.

Die Empfehlungen der nationalen Akademie der Wissenschaften Leopoldina in der dritten Ad-hoc-Stellungnahme vom 13.04.2020 lauteten: „An den Universitäten und Hochschulen sollte das

1 MINT-Fächer: Mathematik, Informatik, Naturwissenschaft und Technik. 
Sommersemester weitgehend als Online/ Home-learning-Semester zu Ende geführt werden. Fließende Übergänge und Mischungen von Fern- und Präsenzunterricht bieten sich an. Voraussetzung sind abgestimmte Lerneinheiten, die digital vermittelt werden" [2].

Anders als in anderen Bildungseinrichtungen, wie z.B. Grundschulen, ist den erwachsenen Studierenden zu unterstellen, dass sie in der Rolle als Beschäftigte der Universität mündig und verantwortungsbewusst für sich und zum Schutz der anderen Studierenden und Beschäftigten handeln können, außerdem bereit sind, auch alternative Lernformen auszuprobieren und weiterzuentwickeln. Demgegenüber stehen Lehrkräfte, die sich ggf. ebenfalls in die Nutzung digitaler Lehrmedien einarbeiten müssen. Auch könnten diese - in Einzelfällen auch Studierende - der Risikogruppe für einen schweren Verlauf von COVID-19 angehören. In der aktuellen Pandemie müssen daher die Hochschulen die Anforderungen in den Lernzielkatalogen und die Vorgaben für das Erreichen des Berufsabschlusses neu betrachten und ggf. Ausnahmeregelungen treffen.

\section{Ziel der Stellungnahme}

Der Artikel hat das Ziel, Wissen zusammenzutragen, wie präventiv die Tätigkeiten in der universitären Lehre für die Lehrenden, die Studierenden und weitere beteiligte Personen möglichst sicher sowie physisch und psychisch wenig belastend im Hinblick auf das COVID-19-Infektionsrisiko gestaltet werden können. Es richtet sich an die Verantwortlichen des Lehrbetriebes; sie haben Arbeits- und Infektionsschutzmaßnahmen zur Minderung des Risikos einer SARS-CoV-2Infektion innerhalb des universitären $\mathrm{Be}$ triebes anzuordnen. Für besonders vulnerable Personengruppen soll nicht nur eine Minderung, sondern eine Minimierung des Infektionsrisikos durch die vorgeschlagenen Maßnahmen erreicht werden. Der Artikel soll dazu beitragen, die erfolgreiche Fortführung und den Abschluss des Studiums unter den neuen Voraussetzungen zu ermöglichen.

\section{Methoden und Lösungsansatz}

Die Autor*innen haben anhand einer internetbasierten Suche nach aktuellen Empfehlungen und Erkenntnissen zu dem Thema und benachbarter Themen durchgeführt, diese zusammengeführt und durch eigene, erfahrungsgestützte Detailüberlegungen $\mathrm{zu}$ spezifischen Lehrformen ergänzt. Die gefundene Anzahl relevanter Internet-basierter Informationen und Literatur $\mathrm{zu}$ diesem Thema wächst aktuell stetig, weswegen die Autor*innen keinen Anspruch auf Vollständigkeit erheben können. Als Lösungsansatz werden die bereits Internet-basiert veröffentlichten und verordneten Hygienemaßnahmen zur COVID-19-Pandemie mit den Arbeitsschutzmaßnahmen entsprechend des STOP-Prinzips kombiniert. Vorliegende Empfehlungen beruhen auf Expert*innenmeinung sowie Vorgaben der entsprechenden Behörden. Es gibt zum derzeitigen Zeitpunkt keine ausreichende quantitative Evidenz für die Wirksamkeit dieser Maßnahmen im Kontext von Infektionen mit SARSCoV-2.

\section{Hygienemaßnahmen}

Ziel der Hygienemaßnahmen ist der Schutz am Arbeitsplatz bzw. im Studium vor der Infektion mit SARS-CoV-2. Daher ist es wichtig, dass jede Person, die dem Bildungsbetrieb an Universitäten und Hochschulen als Beschäftigte/r bzw. Studierende/r angehört, diese allgemeinen Verhaltensregeln kennt. Die Bildungseinrichtungen sollten regelmäßig hierüber informieren und unterweisen (bspw. in Einführungsvorlesungen). Die Hochschulen und Universitäten müssen für die Umsetzung der Kernelemente in allen Bereichen der Hochschulen und Universitäten Sorge tragen. Umfangreiche Informationen stellen sowohl das Robert-Koch-Institut [3, 4] als auch die Bundeszentrale für gesundheitliche Aufklärung [5] zur Verfügung.

Folgende Inhalte sollten die Unterweisungen enthalten:

- Direkten Kontakt meiden

- Einhaltung von mindestens 1,5 m Abstand zu anderen Personen.
- Vermeiden der Berührung von Augen, Nase oder Mund mit ungewaschenen Händen.

- Vermeiden von Begrüßungen durch Umarmungen, Küssen und Händeschütteln.

- Vergrößern des Sprechabstandes.

- Richtiges Händewaschen

- Waschen der Hände häufiger, besonders vor dem Zubereiten und Verzehr von Speisen, nach dem Toilettengang oder dem nach Hause kommen. Auch nach dem Erreichen des Veranstaltungsortes (zum Beginn von Seminaren nach Raumwechsel oder nach Pausen) ist die Reinigung der Hände zu empfehlen. Genügend Waschräume sollten zur Verfügung stehen. Auch in diesen ist die Einhaltung der Abstandsregeln, z. B. durch Reduzierung und Vorgaben zur maximalen Personenzahl in den Wasch- und Toilettenräumen zu empfehlen.

- Die Hände unter fließendes Wasser halten, Seife 20 bis 30 s auch zwischen den Fingern verreiben, dann sorgfältig abspülen und abtrocknen mit Einmaltrockentüchern.

- Hygienisches Husten und Niesen

- Nach dem Husten und Niesen keine Berührung von Gegenständen oder Mitmenschen, Hände waschen.

- Beim Husten oder Niesen größtmöglichen Abstand zu anderen Personen einhalten.

- Husten in die Armbeuge oder Verwendung eines Taschentuches, dieses nur einmal benutzen und sofort entsorgen.

\section{STOP-Prinzip als Leitfaden zur Umstrukturierung}

Das im Arbeitsschutz verankerte STOP ${ }^{2}$ Prinzip ist eine Möglichkeit, die verschiedenen Bereiche der Hochschullehre entsprechend der Gefährdungsbeurteilung strukturiert $\mathrm{zu}$ betrachten; es kann in der Gestaltung einzelner Bereiche helfen, um eine Minderung des SARS-CoV-2-

2 STOP = Substitution, Technische, Organisatorische, Persönliche (Schutzmaßnahmen). 
Infektionsrisikos der Beschäftigten und Studierenden zu erreichen. Das Prinzip gibt die Rangfolge der Schutzmaßnahmen wieder: (1) den Ersatz (Substitution, z.B. durch Online-Angebote) oder den Wegfall nichtdringlicher Aufgaben und Tätigkeiten, (2) die Umsetzung technischer und organisatorischer Maßnahmen zur Einhaltung der Abstandsregeln und außerdem, sollten Letztere aufgrund unvermeidbarer enger Personenkontakte nicht möglich sein, (3) weitere Regelungen zu persönlichen Schutzmaßnahmen.

Die generellen Prinzipien des Infektionsschutzes vor SARS-CoV-2-Infektionen, nämlich

- Kontakte reduzieren und Abstandsregeln einhalten im Unterricht und im Kontakt mit weiteren Beteiligten,

- Reduzierung von Miterkrankungen oder Quarantänefällen durch kleine und feste Teams und

- Tragen persönlicher Schutzkleidung, wenn erforderlich,

können mit dem STOP-Prinzip folgendermaßen umgesetzt werden:

\section{Substitution}

Substituierende Maßnahmen können mögliche infektiöse Kontakte mit anderen Studierenden oder Beschäftigten am effizientesten reduzieren. Dies erfordert die Überprüfung und Entscheidung durch die Leitungsverantwortlichen der Hochschule, ob bisherige Arbeitsweisen durch andere Lehr- und Kommunikationsformen ersetzt werden können. Entsprechende Anordnungen können auch kurzfristig angepasst werden. Maßnahmen der Substitution sind beispielsweise:

- Lernzielkataloge und Lehrangebote überprüfen: Priorisierung der Lehrangebote, die für das weitere Studium und die Abschlüsse essenziell sind.

- Vorlesungen und Seminare durch digitale Angebote ersetzen (Präsentationen mit Tonspur, Webinare, online-Lehrmaterialien für das Selbststudium).

- Bereitstellung von Videokonferenzprogrammen für studentische Diskussionsrunden (mit Schulungen).

Zbl Arbeitsmed 2021 ·1:49-55 https://doi.org/10.1007/s40664-020-00411-9

(c) Der/die Autor(en) 2020

A. M. Preisser · J. Pieter · V. Harth

Präsenzlehre an Universitäten und Hochschulen unter den

Bedingungen der SARS-CoV-2-Pandemie. Empfehlungen zu

technischen, organisatorischen und persönlichen

Schutzmaßnahmen im Rahmen des Arbeitsschutzes

Zusammenfassung

Zur Aufrechterhaltung des universitären Lehrangebotes unter Einhaltung der Abstandsregeln entsprechend des Arbeitsschutzstandards des Bundesministeriums für Arbeit und Soziales (BMAS) sind unter den Bedingungen der COVID-19-Pandemie beim Aufeinandertreffen von Studierenden und Lehrkräften in großen Gruppen (Hörsaal) und kleinen Lehrräumen (Seminare, Praktika) besondere Regelungen und Maßnahmen zu beachten. Der Artikel gibt detaillierte Empfehlungen für die praktische Umsetzung von Schutz- und Präventionsmaßnahmen nach dem im Arbeitsschutz üblichen STOP-Prinzip:
Substitution, Technische, Organisatorische und Persönliche Schutzmaßnahmen. Hierbei werden die Verantwortlichkeiten benannt und auch schwierige Situationen, in denen körperliche Nähe notwendig ist (z.B. Lehre medizinischer Untersuchungen, gemeinsame Laborarbeit, Sport- und Tanzseminare), hinsichtlich der notwendigen Maßnahmen analysiert und Empfehlungen formuliert.

Schlüsselwörter Infektionsgefährdung · Arbeitsschutz · COVID19. Studium · Unterricht

\section{Classroom teaching at universities and colleges under the conditions of the SARS-CoV-2 pandemic. Recommendations on technical, organizational and personal protective measures for occupational health and safety}

\section{Abstract}

In order to maintain university teaching while observing the distance rules in accordance with the occupational health and safety standard of the German Federal Ministry of Labour and Social Affairs (BMAS), special regulations and measures must be observed under the conditions of the COVID-19 pandemic when students and teaching staff meet in large groups (lecture hall) and small teaching rooms (seminars, internships). The article gives detailed recommendations for the practical implementation of protective and preventive measures according to the STOP principle, which is customary in occupational health and safety: substitution, technical, organisational and personal protective measures. Responsibilities are named and also difficult situations where physical proximity is necessary (e.g. teaching medical examinations, joint laboratory work, sports and dance seminars) are analysed with regard to the necessary measures; recommendations are formulated.

Keywords

Risk of transmission - Occupational safety . COVID-19. Study $\cdot$ Teaching
- Überprüfen, ob auch Praktika durch digitale Angebote ersetzt werden können (online-Diskussionsrunden; Demonstrationen über Videos, Bereitstellung von geeigneten Lehrmaterialien in Printform, in digitalen Medien der Bibliotheken oder Angabe geeigneter Internetangebote).

- Präsenzpflichten auf Notwendigkeit überprüfen. Teamsitzungen und Besprechungen per Video- oder Telefonkonferenz.
- Die im Ablauf des Studiums notwendigen Antragstellungen online ermöglichen, anstelle des persönlichen Erscheinens vor Ort.

- Telefonische Beratungen anbieten.

\section{Technische Anpassungen}

- Einrichtung der technischen Voraussetzungen für die Durchführung von Home Teaching and Learning (Software Lizenzen). 


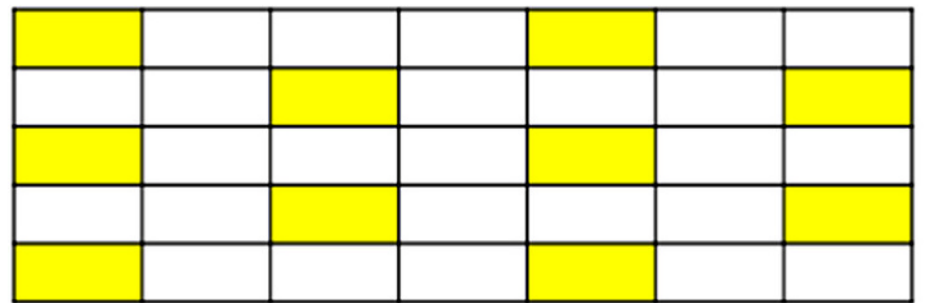

a

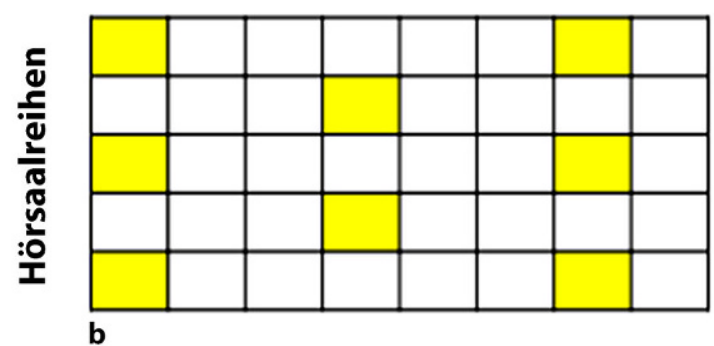

Abb. $1 \Delta$ a, b Sitzanordnungen in den Hörsaalreihen; in jeder Reihe ist jeder 4. oder 6. Platz besetzt, jedoch versetzt zu den benachbarten Reihen: a im Fall von breiten Einzelsitzen $(\mathrm{ca} .90 \mathrm{~cm})$, b im Fall von normalen Einzelsitzen (à $55-60 \mathrm{~cm})$

- Anpassung der räumlichen Gegebenheiten: $z$. B.

- Einbau von Trennwänden, Abstandshaltern, Plexiglastrennscheiben für Praktikumsräume,

- Gewährleistung einer ausreichenden Belüftung,

- Zugang zu Händewasch-Möglichkeiten, ausreichend Seife und Papier,

- Durchgangstüren, soweit möglich, automatisch öffnend/offenlassen,

- personenbezogene Arbeitsplätze, Benutzung von Tastatur, Maus, Arbeitsmaterialien etc. durch mehrere Personen möglichst vermeiden,

- Abstände zwischen Sitzmöglichkeiten in Unterrichtsräumen, Aufenthaltsräumen, Bibliotheken etc. überprüfen (mindestens 1,5-2 m). Aushang der maximalen Personenzahl an der Eingangstür des Unterrichtsraumes.

- Anpassung der Reinigungspläne (Hinweise zu Reinigung und Desinfektion; [3]):

- Intensivierte, häufige Reinigung von Kontaktflächen (Türklinken).

\section{Organisatorische Anpassungen}

- Wiederholte Information und Aufklärung der Mitarbeitenden und Studierenden über die vorgesehenen und einzuhaltenden Maßnahmen zum Infektionsschutz sowie zum Verhalten im Krankheitsfall bzw. bei ersten möglichen Krankheitszeichen (Husten, Halsschmerzen, Fieber, Geschmacksverlust), z. B. durch Aushänge an den Eingängen der Gebäude und in den Toiletten/am Waschbecken bzgl. richtigem Händewaschen, durch Online-Angebote oder Einführungsfolien bei Seminaren etc.

- Reduktion der Gruppengrößen entsprechend der Größe und Möblierung der Unterrichtsräume zur Einhaltung des notwendigen Abstandes von 1,5-2 m.

- Reduktion der Kontaktzeiten zwischen den Mitarbeitenden und den Studierenden bei Raumwechsel z.B. durch zeitliche Entzerrung der Stundenpläne, exakte Absprachen bezüglich Nutzungszeiten von Räumlichkeiten.

- Vorgabe der Wechselwege (z. B. nur von einer Seite in die Reihe eintreten, zur anderen Seite aus der Reihe austreten), Kennzeichnung mit Richtungspfeilen in den Aufgängen und Abgängen, Trennung der Verkehrswege durch Bodenmarkierungen.

- Kennzeichnung der zu nutzenden Sitzplätze in den Hörsälen, jede Reihe nutzen (ca. jeder 4. Bis 6. Platz je nach Sitzbreite, versetzt zur vorherigen Reihe, somit Abstandswahrung nach schräg vorne bzw. hinten von je 1,5-2 m; - Abb. 1); auch, damit im Falle eines Austretens möglichst wenige weitere Studierende mit aufstehen und austreten müssen.

- Gewährung von Home Learning \& Teaching, wenn möglich.

- Einteilen von festen Studierendengruppen und Betreuungspersonen, damit im Erkrankungsfall nur eine begrenzte Zahl an Kontaktpersonen in Frage kommt und die Infektionsermittlung effektiv durchgeführt werden kann.
- Festlegung des weiteren Vorgehens (Dienstanweisung o. Ä.) bei Auftreten eines COVID-19-Erkrankungsfalls in der Hochschuleinrichtung mit Benennung fester Ansprechpartner*innen für Hygienefragen sowie für die Organisation und Umsetzung der Maßnahmen. Zur Vorbereitung hierauf gehört auch das Führen von Teilnehmerlisten und ggf. Erfassung der privaten Telefonnummern (oder Sicherstellung einer Einsicht in die Kontaktdaten durch den Krisenmanager auch am Wochenende oder abends außerhalb von Sekretariatsöffnungszeiten), sodass betroffene Personen schnell informiert werden können. Teilnehmerlisten sind datenschutzkonform zu erstellen und aufzubewahren. Ein entsprechendes Konzept ist erforderlich.

- Einhalten der räumlichen Trennung der einzelnen Teams auch während der Pausenzeiten.

\section{Persönliche Verhaltensregeln}

- Einhalten der Abstandsregeln, auch während der Pausenzeiten.

- Husten- und Niesetikette (in Ellenbeuge) ist einzuhalten.

- Mitarbeitende und Studierende mit Symptomen einer akuten Atemwegserkrankung sollen der Universität oder der Hochschule fernbleiben. 


\section{Besondere Lehrformen und Arbeitsräume}

\section{Praktika (Labore, technische Räume)}

In diesen Lehrveranstaltungen sind im Wesentlichen die Abstandsregeln einzuhalten, wie oben beschrieben. Dies erfordert kleine Gruppen; die ursprünglichen Gruppengrößen müssen entsprechend aufgeteilt und die Veranstaltungshäufigkeit entsprechend erhöht werden. Die begrenzten Kapazitäten von Räumen und Dozierenden können folgend dazu zwingen, dass andere Praktika aktuell weiterhin nicht in Präsenzform angeboten werden können. Zutrittszeiträume sind festzulegen; die Pausen sollten umschichtig erfolgen. Diese Umsetzungen, die erhebliche Auswirkungen auf den Lehrbetrieb haben, müssen von der Hochschulleitung vorgegeben werden.

Die Hochschulleitung muss zudem Regelungen für den Zugang und das Verlassen der Räume treffen, um das Abstandhalten von Einzelpersonen zu ermöglichen. Auch Gänge zum Händewaschen, zu Toiletten und evtl. Pausen können nur mit Abstand erfolgen. Zusätzliche Mund-Nasen-Bedeckungen sind zu erwägen, wenn der Abstand nicht zuverlässig eingehalten werden kann.

\section{Unterricht mit engem Kontakt unter den Studierenden}

Um in medizinischen Untersuchungskursen ohne Patient*innen, Kursen in Schauspiel und Tanz oder in Sportkursen gegenseitige Infektionen $\mathrm{zu}$ vermeiden, müssen alle Beteiligten eine MundNasen-Bedeckung tragen. Bei Unterrichtsformen, in denen Masken den Unterrichtserfolg behindern würden (z. B. Schauspiel, Sportkurse), muss der Verzicht auf Atemschutz erwogen werden. Bei einem Verzicht auf Atemschutz sollten kleine Gruppen (ca. max. 6 Personen) zusammenarbeiten; die Personen sollten zwischen den Teams nicht wechseln.
Unterricht mit Kontakt zu anderen Personen oder Berufsgruppen, die nicht der Universität angehören

Lehrende und Studierende sollten den Abstand von $>1,5 \mathrm{~m}$ konsequent beibehalten, sogar möglichst eher vergrößern. Wir empfehlen das Tragen von MundNasen-Schutz. Auch bei Einbeziehung von Schauspielpatient ${ }^{*}$ innen, z. B. in der Vermittlung von Kommunikationstechniken, soll der Abstand bei den sprechenden Personen so groß wie möglich sein, ein Sprechabstand von 2-3 $\mathrm{m}$ wird von uns empfohlen. Sollte die Abstandsregel nicht zuverlässig und durchgängig umgesetzt werden können (jedoch keine engen Körperkontakte erwartet werden), empfehlen wir auch hier eine Mund-NasenBedeckung für alle Beteiligten (Schauspieler*innen, Studierende und Lehrende).

\section{Unterricht mit engem Kontakt zu Personen, die nicht der Universität angehören und/oder als besonders gefährdet für einen schweren Verlauf einer COVID-19- Infektion eingestuft werden}

Diese Unterrichtsform muss wegen des besonderen Risikos für die beteiligten Drittpersonen, i.d.R. Patient ${ }^{*}$ innen in den medizinischen Studiengängen, minimiert werden und kann evtl. in der aktuellen Situation auch gar nicht zugelassen werden. Insbesondere $\mathrm{Pa}-$ tient ${ }^{\star}$ innen mit dem Risiko, im Falle einer Infektion einen schweren Covid19-Krankheitsverlauf zu erleiden, sind zu schützen. Die Einschätzung richtet sich nach den aktuellen Erkrankungszahlen und den Einschränkungen für andere Besucher und Angehörige. Diese Einschätzung obliegt den behandelnden Ärzt ${ }^{*}$ innen. Der Besuch von Angehörigen stationärer Patient ${ }^{*}$ innen hat i.d.R. Vorrang vor der studentischen Lehre. Sollte Unterricht mit Patient ${ }^{*}$ innen erfolgen müssen, die ein hohes Risiko für einen schweren Verlauf haben, sind den Patient*innen zum Eigenschutz FFP2Masken anzubieten. Alle anderen Patient ${ }^{*}$ innen sollen medizinischen MundNasen-Schutz (MNS) tragen [6]; die geltenden Hygieneregeln des Universitäts- oder Lehrkrankenhauses sind zu beachten. Für die Studierenden sind die konsequente Händereinigung und -desinfektion sowie das Tragen von MNS und regelmäßig gereinigter Schutzkleidung obligat. Ob das korrekte Tragen des medizinischen MNS hier nicht nur dem Fremdschutz dient, sondern auch den Träger vor der Aufnahme von Tröpfchen oder Spritzern über Mund oder Nase, z.B. aus dem Nasen-Rachen-Raum des Gegenübers schützt (Eigenschutz; [6-8]), oder ob lediglich partikelfiltrierende Halbmasken (FFP-Masken) ausreichenden Eigenschutz bieten [9, 10], ist weiterhin in Diskussion. Einfache Stoffmasken bieten jedoch einen geringeren Eigenschutz als MNS und sind hier nicht zu empfehlen [6]. Unterricht mit infektiösen, an COVID19 erkrankten Patient*innen ist nicht möglich.

Zahnmedizinischer Unterricht am Patienten erfordert einen höheren Schutz auf Seite der Studierenden, da die Patient ${ }^{*}$ innen selbstverständlich keine Mund-Bedeckung tragen können. Außerdem ist eine vermehrte Aerosolbildung bei zahnärztlichen Behandlungen möglich. Dies erfordert prinzipiell den Einsatz entsprechender Absaugtechnik [10]. Die Studierenden sollen Schutzkleidung und FFP2-Masken tragen, ergänzt durch einen Gesichtsschild, der zusätzlich vor Spritzern schützt, die mit Patient ${ }^{*}$ innen-Sekreten vermischt sein könnten. Diese doppelte Schutzmaßnahme (Atemschutzmaske plus Gesichtsschild) soll auch einen zusätzlichen Schutz der Patient ${ }^{\star}$ innen bei Unterschreitung des Abstandes von $1,5 \mathrm{~m}$ im Sinne des Fremdschutzes bewirken. Infektiöse oder entsprechend verdächtige Personen dürfen von den Studierenden nicht behandelt werden.

Auch bei diesen Unterrichtsformen sind die Abstandsregeln zwischen den direkt beteiligten Personen und den momentan nicht direkt Beteiligten möglichst einzuhalten. Eine Unterschreitung der Distanz von 1,5 m zu den Risikopersonen und Patient ${ }^{*}$ innen sollte nur möglichst kurzzeitig und von nur wenigen Personen erfolgen. 


\section{Prüfungen}

Wenn Präsenzprüfungen aufgrund der Prüfungsordnung nicht durch OnlineFormate oder analoge Prüfungsformate (z.B. Hausarbeiten) ersetzt werden können, so ist hier im Wesentlichen sicherzustellen, dass die Abstandsregeln eingehalten werden. Für schriftliche Prüfungen sind große Räumlichkeiten, z. B. Hörsäle, notwendig. Damit die einzelnen Reihen im Hörsaal nicht zu voll besetzt werden und im Falle eines Toilettengangs nicht zu viele Menschen in der Reihe aufstehen müssen (ein aneinander Vorbeischieben ist aufgrund der Abstandsregel nicht möglich), sollte nur ca. jeder 4 . oder 6. Platz besetzt werden. Hierdurch kann ein Abstand zu der schräg davor bzw. schräg dahinter sitzenden Person von ca. 1,5-2 m eingehalten werden; • Abb. 1. Beim Einlass, Toilettengang und Verlassen des Prüfungsraums ist die Einhaltung der Abstandsregel und Kennzeichnung der Verkehrswege (s. oben) sicherzustellen. Aufgrund der hohen Personenzahl mit möglicherweise kurzzeitigen Unterschreitungen der Abstandsregel sollten beim Ein- und Austreten Mund-NasenBedeckungen von Prüflingen und Aufsichtspersonal getragen werden.

Prüfungen, die ein Unterschreiten des Abstandes von 1,5 m erfordern, sind mit den Schutzmaßnahmen wie die entsprechenden Unterrichtseinheiten zu gestalten (s. oben).

\section{Exkursionen}

An vielen Universitäten gilt aktuell noch ein generelles Dienstreiseverbot, welches auch die Lehrtätigkeit umfasst. Nach voraussichtlicher Lockerung dieser Regelungen sind die Vorgaben des Reiseverkehrs (Bahn, Flug) und die der zu besuchenden Institution $\mathrm{zu}$ beachten. Sollen wissenschaftliche Exkursionen außerhalb der BundesrepublikDeutschland stattfinden, sind die Reisewarnungen des Auswärtigen Amtes sowie die nationalen Bestimmungen des Ziellandes zu beachten [11].

\section{Umsetzung}

Die Vermittlung der genannten Maßnahmen kann über öffentliche Empfehlun- gen, Publikationen oder Leitlinien erfolgen. Die Umsetzung dieser Maßnahmen des Arbeitsschutzes in der Universität oder Hochschule ist durch die entsprechende Hochschulleitung, Präsidium bzw. die Geschäftsführung sicherzustellen. Diese sollten die Beratung durch die Sicherheitsfachkraft, die Hygienefachkraft und die Betriebsärzt*innen in Anspruch nehmen. Entsprechende Unterweisungen der Mitarbeiter*innen sind zu empfehlen. Die den Maßnahmen zugrunde zu legende Gefährdungsbeurteilung (nach ArbSchG [12]) kann mit Hilfe der Muster-Gefährdungsbeurteilung für den Interimsbetrieb der Hochschulen in der Corona-Pandemie [13] erstellt werden.

Auch die psychische Belastung der Beschäftigten und Studierenden ist in Bezug auf die veränderten Umstände zu berücksichtigen $[14,15]$. Die Kommunikation der Hochschule bezüglich der betrieblichen Maßnahmen sollte klar und gut verständlich sein. Wichtige Informationen sollten allen Personen, die von den Änderungen betroffen sind, zugänglich sein (Aushänge, E-Mail-Verteiler etc.). Wichtige Ansprechpartner sollten benannt und die Kontaktdetails ebenfalls gut zugänglich sein (z.B. online). Wir empfehlen, bei Bedarf Schulungen z.B. zu neuen digitalisierten Arbeitsformen, Hygienemaßnahmen, psychischer Gesundheit und der richtigen Verwendung von Schutzkleidung durchzuführen (Merkzettel, Videoschulungen, E-Learning-Angebote, telefonische Beratungen bei individuellen Fragen).

Bei der Festlegung und Umsetzung der Maßnahmen sollten auch die Studierenden- und Arbeitnehmervertretungen hinzugezogen und ihre Umsetzungsideen, Sorgen und Fragen berücksichtigt werden [16].

Zusätzlich sollte das individuelle Risiko der Beschäftigten (Lehrenden und Studierenden) für einen schweren Verlauf einer SARS-CoV-2-Erkrankung im Rahmen einer arbeitsmedizinischen Beratung erwogen werden. Sowohl die lehrenden Beschäftigten als auch die Studierenden genießen im Falle einer Infektion den gesetzlichen Versicherungsschutz der Unfallkassen der Länder. Die Betriebsmediziner*innen können eine Be- ratung für Zugehörige von Risikogruppen oder im Erkrankungsfall auch telefonisch anbieten. Die Zugehörigkeit zu einer Risikogruppe kann dazu führen, dass eine Umsetzung an einen Arbeitsplatz oder die Bereitstellung von individuellen Lehrformen mit geringerem Risiko für eine Ansteckung (Home Office, E-Learning und Teaching) erforderlich wird. Studierende mit erhöhtem Risiko eines schweren COVID-19-Krankheitsverlaufes sollen durch die eingeschränkte Teilnahme an Lehrveranstaltungen mit notwendiger Präsenz keinen Nachteil in ihrem Studium erfahren.

Weitere Informationen zu Risikogruppen finden sich auf der Homepage des Robert-Koch-Instituts [17] sowie in den Policy-Briefen „Müssen ältere Beschäftigte dem Arbeitsplatz fernbleiben?" von Seidler und Petereit-Haack [18] sowie „Beschäftigte mit erhöhtem Krankheitsrisiko" von Angerer, KaifiePechmann und Tautz [19].

\section{Fazit und Empfehlungen}

Im Hinblick auf eine weitere Öffnung des Hochschulbetriebes in Bezug auf Präsenzzeiten und praktische Erfahrungen müssen präventive Maßnahmen etabliert werden, um eine Verbreitung der COVID-19-Infektion innerhalb der Lehrbetriebe $\mathrm{zu}$ verlangsamen und Risikogruppen zu schützen. Eine Vielzahl an Maßnahmen der Risikominderung und des Infektionsschutzes im Rahmen des Arbeitsschutzes sind umsetzbar. Die Maßnahmen können stufenweise entsprechend des STOP-Prinzips aufgebaut werden und sich an den folgenden Prinzipien orientieren: konsequente Hygienemaßnahmen, Vermeidung nicht notwendiger Kontakte, Kontaktreduzierung und Reduzierung der Anzahl eventueller Quarantänefälle, Einhalten der Abstandsregelung und der Situation angepasstes Tragen von persönlicher Schutzausrüstung. 


\section{Korrespondenzadresse}

PD Dr. med. Alexandra M. Preisser

Zentralinstitut für Arbeitsmedizin und Maritime Medizin (ZfAM), Universitätsklinikum Hamburg-Eppendorf

Seewartenstraße 10, 20459 Hamburg,

Deutschland

a.preisser@uke.de

Danksagung. Die Autor*innen danken P. Angerer, E. M. Bitzer, U. Bolm-Audorff, T. Fischer und K. Geffert für die kritische Durchsicht des Manuskriptes vor der Erstveröffentlichung im Juni $2020 \mathrm{im}$ Rahmen des Kompetenznetzes Public Health COVID-19.

Funding. Open Access funding enabled and organized by Projekt DEAL.

\section{Einhaltung ethischer Richtlinien}

Interessenkonflikt. A.M. Preisser, J. Pieter und V. Harth geben an, dass kein Interessenkonflikt besteht.

Für diesen Beitrag wurden von den Autoren keine Studien an Menschen oder Tieren durchgeführt. Für die aufgeführten Studien gelten die jeweils dort angegebenen ethischen Richtlinien.

Open Access. Dieser Artikel wird unter der Creative Commons Namensnennung 4.0 International Lizenz veröffentlicht, welche die Nutzung, Vervielfältigung, Bearbeitung, Verbreitung und Wiedergabe in jeglichem Medium und Format erlaubt, sofern Sie den/die ursprünglichen Autor(en) und die Quelle ordnungsgemäß nennen, einen Link zur Creative Commons Lizenz beifügen und angeben, ob Änderungen vorgenommen wurden.

Die in diesem Artikel enthaltenen Bilder und sonstiges Drittmaterial unterliegen ebenfalls der genannten Creative Commons Lizenz, sofern sich aus der Abbildungslegende nichts anderes ergibt. Sofern das betreffende Material nicht unter der genannten Creative Commons Lizenz steht und die betreffende Handlung nicht nach gesetzlichen Vorschriften erlaubt ist, ist für die oben aufgeführten Weiterverwendungen des Materials die Einwilligung des jeweiligen Rechteinhabers einzuholen.

Weitere Details zur Lizenz entnehmen Sie bitte der Lizenzinformation auf http://creativecommons.org/ licenses/by/4.0/deed.de.

\section{Literatur}

1. Bundesministerium für Arbeit und Soziales (2020) SARS-CoV-2-Arbeitsschutzstandard, Stand: 16.04.2020. https://www.bmas.de/DE/ Presse/Pressemitteilungen/2020/einheitlicherarbeitsschutz-gegen-coronavirus.html. Zugegriffen: 16. Apr. 2020

2. Nationale Akademie der Wissenschaften Leopoldina (2020) Leopoldina-Stellungnahmen zur Coronavirus-Pandemie; Stand: 13.04.2020. https://www.leopoldina.org/ publikationen/detailansicht/publication/ leopoldina-stellungnahmen-zur-coronaviruspandemie-2020/.Zugegriffen: 13.Apr. 2020

3. Robert-Koch-Institut (2020a) Hinweise zu Reinigung und Desinfektion von Oberflächen außerhalb von Gesundheitseinrichtungen im Zusammenhang mit der COVID-19-Pandemie; Stand 04.04.2020. https://www.rki.de/DE/Content/ InfAZ/N/Neuartiges_Coronavirus/Reinigung_ Desinfektion.html?nn=13490888. Zugegriffen: 21. Apr. 2020

4. Robert-Koch-Institut (2020b) Mund-Nasen-Bedeckung im öffentlichen Raum als weitere Komponente zur Reduktion der Übertragung von COVID-19. Strategie-Ergänzung zu empfohlenen Schutzmaßnahmen und Zielen (3. Update) Epid Bull 19:3-5. https://www.rki.de/DE/Content/ Infekt/EpidBull/Archiv/2020/Ausgaben/19_20. pdf?_blob=publicationFile. Zugegriffen: 29 . Juni 2020

5. Bundeszentrale für gesundheitliche Aufklärung (2020) Virusinfektion - Hygiene schützt!; Stand: 27.07.2020. https://www.infektionsschutz. de/fileadmin/infektionsschutz.de/Downloads/ 200520_BZgA_Atemwegsinfektion-Hygiene_ schuetzt_A4.pdf.Zugegriffen:29.Juni 2020

6. Dellweg D, Lepper PM, Nowak D, Köhnlein T, Olgemöller U, Pfeifer M (2020) Stellungnahme der DGP zur Auswirkung von Mund-Nasenmasken auf den Eigen- und Fremdschutz bei aerogen übertragbaren Infektionen in der Bevölkerung. Pneumologie 74(06):331-336. https://doi.org/10. 1055/a-1175-8578

7. Robert-Koch-Institut (RKI) (2020c) Empfehlungen desRKIzuHygienemaßnahmen im Rahmen derBehandlung und Pflege von Patienten miteiner Infektion durch SARS-CoV-2; Stand: 05.06.2020. https:// www.rki.de/DE/Content/InfAZ/N/Neuartiges_ Coronavirus/Hygiene.html. Zugegriffen: 29. Juni 2020

8. Kämpf $D$, Bolm-Audorff U, Petereit-Haack G, Stranzinger J (2020) COVID-19 und Arbeitsschutz im Gesundheitswesen. Technische, organisatorische und persönliche Schutzmaßnahmen zur Verhütung einer Infektion mit SARS CoV-2 bei Beschäftigten im Gesundheitswesen (ohne Alten- und Pflegeeinrichtungen). 2. überarbeitete Version. https://www.public health-covid19.de/images/2020/Ergebnisse/ COVID-19_UAG_Arbeitsschutz_Handreichung_ Gesundheitswesen_2020jun24_Version2-final. pdf. Zugegriffen:29. Juni 2020

9. Bundesinstitut für Arzneimittel und Medizinprodukte: Hinweise des BfArM zur Verwendung von Mund-Nasen-Bedeckungen (z. B. selbst hergestellten Masken, "Community- oder DIY-Masken"), medizinischen Gesichtsmasken sowie partikelfiltrierenden Halbmasken (FFP1, FFP2 und FFP3) im Zusammenhang mit dem Coronavirus (SARS-CoV-2 / Covid-19) https://www.bfarm.de/SharedDocs/ Risikoinformationen/Medizinprodukte/DE/ schutzmasken.html [24.09.2020]

10. Bundesanstalt für Arbeitsmedizin und Arbeitsschutz (BAuA) (2018) Technische Regeln für Biologische Arbeitsstoffe: TRBA 250 Biologische Arbeitsstoffe im Gesundheitswesen und in der Wohlfahrtspflege. https://www.baua. de/DE/Angebote/Rechtstexte-und-TechnischeRegeln/Regelwerk/TRBA/pdf/TRBA-250.pdf? blob=publicationFile \&v=4. Zugegriffen: 29 . Juni 2020

11. Deutsche Gesetzliche Unfallversicherung e.V. (DGUV) (2020) Coronavirus (SARS-CoV-2) -
Empfehlungen für Hochschulen und Forschungseinrichtungen; Stand 22.04.2020. https://www. dguv.de/corona-bildung/hochschulen/index.jsp. Zugegriffen: 2. Juli 2020

12. Arbeitsschutzgesetz (ArbSchG), vom 7. August 1996 (BGBI.IS. 1246), zuletzt durch Artikel 113 des Gesetzes vom 20. November 2019 (BGBI. IS. 1626) geändert. https://www.gesetze-im-internet.de/ arbschg/_5.html [29.06.2020]

13. AGUM e. V. in Zusammenarbeit mit dem Sachgebiet Hochschulen und Forschungseinrichtungen der Deutschen Gesetzlichen Unfallversicherung (DGUV). Muster-Gefährdungsbeurteilung für den Schutz gegen die Ausbreitung von Krankheitserregern und die Aufrechterhaltung des Interimsbetriebs der Hochschulen, gültig für die Feststellung einer epidemischen Lage von nationaler Tragweite (\$5(1) IfSG): aktuell Coronavirus SARSCoV-2; Stand: 27. Apr. 2020. https://www.dguv. de/medien/inhalt/gbu_corona_agum_2020_04_ 23_final.docx [02.07.2020]

14. Dragano N, Diebig M, Faller G, Honnings A, Hoven H, Lang J, Loerbroks A, Lunau T (2020) Arbeitsverdichtung, Angst vor Infektionen und Co.: Management psychischer Arbeitsbelastungen während der COVID-19 Pandemie. https://www.public-health-covid19.de/images/ 2020/Ergebnisse/Handreichung_Management_ psychischer_Arbeitsbelastungen_COVID-19 V01_10_06_2020.pdf.Zugegriffen:2.Juli 2020

15. Riedel-Heller SG, Röhr S, Seidler A, Apfelbacher C (2020) Psychosoziale Folgen von Isolations-und Quarantänemaßnahmen: Womit müssen wir rechnen? Was können wir dagegen tun? Ergebnisse einer systematischen Literaturrecherche („Rapid Review“). https:// www.public-health-covid19.de/images/2020/ Ergebnisse/Policy_Brief_Psychosoziale_Folgen von_Isolation_30042020_final.pdf. Zugegriffen: 2. Juli 2020

16. U.S. Department of Labor Occupational Safety and Health Administration. Guidance on Preparing Workplaces for COVID-19. OSHA 3990-03 2020. https://www.osha.gov/Publications/OSHA3990. pdf. Zugegriffen: 2 . Juli 2020

17. Robert-Koch-Institut (2020d) Informationen und Hilfestellungen für Personen mit einem höheren Risiko für einen schweren COVID-19Krankheitsverlauf; Stand: 13.05.2020. https:// www.rki.de/DE/Content/InfAZ/N/Neuartiges_ Coronavirus/Risikogruppen.html?nn=13490888. Zugegriffen: 2. Juli 2020

18. Seidler A, Petereit-Haack G, Riedel-Heller SG, Apfelbacher C, Romero-Starke K, Kämpf D, Harth V Angerer $P$ (2020) Müssen ältere Beschäftigte dem Arbeitsplatz fernbleiben? Ergebnisse einer systematischen Literaturrecherche („Rapid Scoping Review"); Stand: 24.04.2020. https://www.publichealth-covid19.de/images/2020/Ergebnisse/ 2020_04_23_Fact_Sheet_Auswirkungen_auf Itere Beschftigte V3.pdf.Zugegriffen: 2. Juli 2020

19. Angerer P, Kaifie-Pechmann A, Tautz A (2020) Beschäftigte mit erhöhtem Krankheitsrisiko. Umgang mit Risikogruppen für schwere Krankheitsverläufe an Arbeitsplätzen oder in Tätigkeiten mit erhöhtem COVID-19 Infektionsrisiko; Stand: 30.04.2020. https://www.public-health-covid19. de/images/2020/Ergebnisse/Beschaftigte_mit_ erhohtem_Krankheitsrisiko_Update_V2_AKPA_ Neues markiert_OK_PA_AK_LG_finale_Version1.pdf.Zugegriffen: 2 . Juli 2020 